\title{
Ureteral Valve Masquerading as Obstructive Megaureter
}

\section{Sushmita Bhatnagar ${ }^{1,2^{*}}$}

${ }^{1}$ Department of Pediatric Surgery, B.J. Wadia Hospital for Children, Parel, Mumbai, India

${ }^{2}$ Bombay Hospital Institute of Medical Sciences, Mumbai, India

\begin{abstract}
Primary obstructed megaureter is a common urological condition in the pediatric age group. It is one of the differentials for congenital anomalies of the kidney and urinary tract that include a myriad of structural anomalies of the urinary tract. The obstructive conditions are usually managed conservatively unless there is deterioration in renal function, repeated urinary tract infection, or any other symptom such as abdominal pain on ipsilateral side and hypertension. Presented here is a case of left lower ureteral valve that was diagnosed as primary obstructed megaureter with pain in abdomen and reduced ipsilateral renal function wherein ureteral valve was detected incidentally intraoperatively. Excision of the valve with end to end uretero-ureteral anastomoses over a D-J stent, without resorting to ureteric reimplantation was successful in relieving the obstruction and hydronephrosis.
\end{abstract}

Keywords: Primary obstructed megaureter; Ureteral valves; Ureteroureteral anastomoses

\section{Introduction}

Valve in the ureter is a rare cause of obstructive uropathy and congenital anomaly of the urinary tract. In the presence of hydronephrosis and hydroureter, the two most common differential diagnoses are vesicoureteric reflux (VUR) and obstruction at vesicoureteric junction, which can be easily differentiated by a micturating cystourethrogram (MCU).

It is generally assumed that in the absence of VUR, obstruction at the vesicoureteric junction is the cause of hydroureteronephrosis as obstructed megaureter is a common condition. Apart from the ureteric orifice, obstructive pathologies of the ureter also present in the same manner and are difficult to diagnose on routine investigations, especially when the ureter is dilated and tortuous. Hence, ureteric valves masquerade as either pelviureteric junction or vesicoureteric junction obstruction, depending on the level of ureteric anomaly.

The conditions that cause intrinsic ureteral obstruction between the pelviureteric and the ureterovesical junction in children are strictures of ureter, either primary (congenital) or secondary (postoperative/ postintervention, stones, tuberculosis, tumors), ureteric polyps, ureteric valves (upper, mid, or lower ureter), stenosis, or diverticula. Unless detailed investigations such as CT-Urography/MR-Urography/ Retrograde ureterogram is done, the exact diagnosis is elusive.

Presented here is one such child who was diagnosed as obstructed megaureter and taken up for ureteric reimplantation surgery but was found to have ureteral valve intraoperatively.

\section{Case Presentation}

A 2-year-old male child presented with pain in abdomen and crying during micturition. There was no history of urinary tract infections. The general as well as systemic examination findings did not show any abnormality. Results of the blood, urine, and culture were negative. Abdominal ultrasound showed a normal right kidney and ureter, a hydronephrotic left kidney measuring $8.2 \times 3.1 \times 4.2 \mathrm{~cm}$, hydroureter with uniform dilatation measuring $12 \mathrm{~mm}$, and a normal bladder. The MCU was normal; there was no evidence of reflux; and intravenous pyelogram (IVP) showed mild hydronephrosis, massively dilated and tortuous left lower ureter, and moderate hydroureter in the mid and upper ureter (Figure 1). Diethylene-triamine-penta-acetic acid (DTPA) scan showed delayed excretion of dye on the left side with $t_{1 / 2}$ more than $20 \mathrm{~min}$. Dimercaptosuccinic acid (DMSA) scan showed reduced function on left side, the differential being $29.7 \%$, and absence of scars.

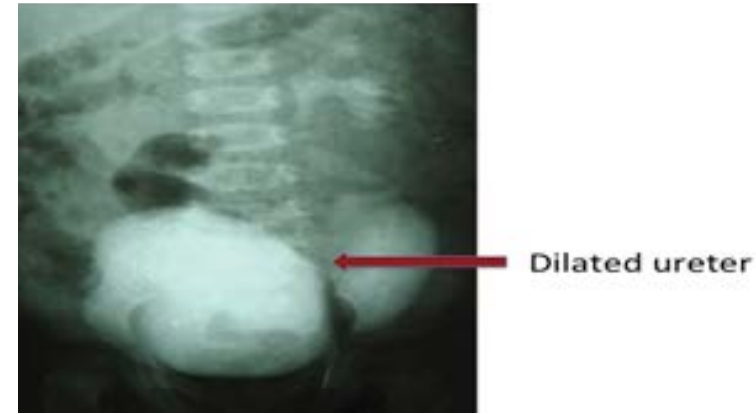

Figure 1: IVP showing dilated ureter in the entire course.

A diagnosis of left obstructed megaureter was made and the child was posted for elective surgery. Intraoperatively, the left ureter was mobilized extravesically to find a normal ureterovesical junction and a normal distal ureter up to $5 \mathrm{~cm}$ from the ureterovesical junction proximal to which ureter was dilated. A $3 \mathrm{~cm}$ longitudinal incision was centered over the narrow part of the ureter to find a ureteric valve, with a thick walled dilated proximal ureter and normal distal ureter (Figure 2). The segment of ureter was excised leaving the distal ureter and ureterovesical junction intact, and a spatulated ureterouretric anastomosis was carried out over a D-J stent. Postoperative course was uneventful, and the D-J stent was removed after a month. The patient was followed up for urinary tract infection every month for first 3 months. After 3 months, an ultrasonography and an MCU were carried out, which did not show any abnormality. Thereafter, the patient was followed up every 3 months till 1 year post operation and then every year for the next 2 years. The child remained well, the renal function was stable, and no hydronephrosis, hydroureter or vesico-ureteric reflux was observed during the 3-year follow-up.

*Corresponding author: Sushmita Bhatnagar, Department of Pediatric Surgery B.J. Wadia Hospital for Children, 56/B, Venus Apartments, Worli Sea Face, Worli, Mumbai 400 018, India, Tel: 9869013797; E-mail: sushmitabh@gmail.com, bhatnagar_s1206@yahoo.co.in

Received December 06, 2016; Accepted January 05, 2017; Published January 12, 2017

Citation: Bhatnagar S (2017) Ureteral Valve Masquerading as Obstructive Megaureter. Surgery Curr Res 7: 285. doi: 10.4172/2161-1076.1000285

Copyright: ( 2017 Bhatnagar S. This is an open-access article distributed under the terms of the Creative Commons Attribution License, which permits unrestricted use, distribution, and reproduction in any medium, provided the original author and source are credited. 


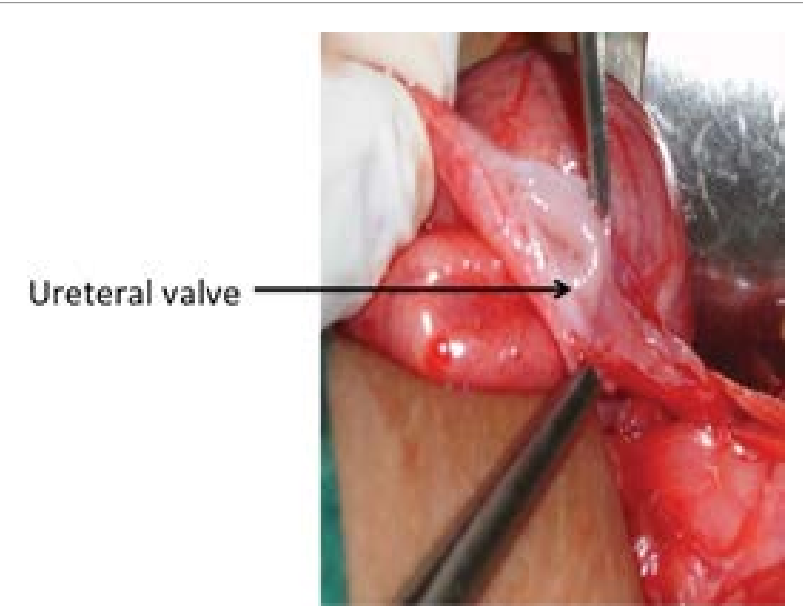

Figure 2: Vertical incision on ureter showing ureteral valve with dilated and thick walled proximal ureter and normal distal ureter.

\section{Discussion}

Congenital ureteral valves are a rare cause of ureteral obstruction in children first described by Wolfer in 1887, approximately just about 100 cases being reported so far [1]. The criteria for definitive description of this condition as described by Wall et al. [2] are as follows:

\section{Clinical criteria:}

a. Dilated ureter above and normal ureter below the valve indicating obstruction.

b. No other evidence of mechanical or functional obstruction.

2. Histological criteria: Presence of transverse folds of the ureteric mucosa containing bundles of smooth muscle fiber.

The most common presentation of ureteral valves is urinary tract infection, pain in abdomen, and rarely, a lump in abdomen due to hydronephrosis. Our patient came with recurrent pain in abdomen for which he underwent an abdominal ultrasonography that showed hydronephrosis and on further evaluation he was diagnosed with vesicoureteric junction obstruction.

Ureteral valve is difficult to diagnose preoperatively unless specific tests such as contrast CT/MR urography, retrograde, pyelography, or ureteroscopy are carried out. Generally, ultrasonography and IVP with renal scan are used to evaluate the anatomy and renal function and to establish the indication of surgical intervention. Whether or not MRU (magnetic resonance urogram) should be performed in all patients of kidney and urinary tract anomalies needs to be determined.
In children, vesicoureteric reflux could be associated with ureteric valves, which makes the diagnosis even more difficult. Other associated urinary tract abnormalities are renal dysgenesis, ureteral atresia, and urethral duplication.

Valves in the upper ureter present as obstruction at pelviureteric junction and can be accurately diagnosed with retrograde pyelography. On one hand, this condition is difficult to diagnose with routine urologic investigations and on the other hand, antenatal detection of ureteric valves by ultrasound has been reported [3].

Management of children with ureteric valves largely involves surgical intervention wherein the segment of ureter with the valve is excised and ureteroureteric (for mid and lower ureter) and pyeloureteral (for upper ureter) anastomoses are performed [4]. Other methods involve endoscopic incision of the valve or use of Holmium laser via ureteroscope to ablate ureteric valve [5].

In cases where the valve is close to the bladder as well as with associated VUR, excision of the lower ureter and ureteric reimplantation into the bladder is desirable. If sufficient length of the lower ureter is present distal to the valve, there is no compulsion to disturb the normal vesicoureteric junction and reimplant the ureter, as was done in our case with excellent postoperative and long-term outcome.

\section{Conclusion}

In the present era with advanced diagnostic technology, children with obstructive uropathy should be investigated in detail with MRU to establish an accurate preoperative diagnosis for optimum management, as the management of the condition could be inappropriate if diagnosis is missed intra-operatively.

\section{References}

1. Montoya-Chinchilla R, Guirao-Piñera MJ, Nortes-Cano L (2014) Uretera valves: Literature review and description of 4 new cases. An Pediatr (Barc) 80: 51-54.

2. Wall B, Wachter $E$ (1952) Congenital ureteric valve: its role as a primary obstructive lesion: Classification of the literature and report of an authentic case. J Urol 68: 684-690.

3. Reinberg Y, Aliabadi H, Johnson P, Gonzalez R (1987) Congenital ureteral valves in children: Case report and review of the literature. J Pediatr Surg 22: 379-381.

4. Rabinowitz R, Kingston TE, Wesselhoeft C, Caldamone AA (1998) Ureteric valves in children. Urology 51: 7-11.

5. Singh SK, Wadhwa $P$ (2006) Ablation of diaphragmatic annular ureteral valve with holmium laser. Int Urol Nephrol 38: 157-159. 